LETTER TO JMG

\title{
Screening for genomic rearrangements of the $M M R$ genes must be included in the routine diagnosis of HNPCC
}

\author{
F Di Fiore, F Charbonnier, C Martin, S Frerot, S Olschwang, Q Wang, C Boisson, M-P Buisine, \\ $M$ Nilbert, A Lindblom, T Frebourg
}

J Med Genet 2004;41:18-20

l hereditary non-polyposis colorectal cancer (HNPCC), the most common form of inherited colorectal cancer, detection of the causal alteration of the mismatch repair $(M M R)$ gene involved is essential for proper management of the families. This will allow the identification of relatives with high risk for colorectal or endometrial cancer, who require the appropriate screening and, conversely, will avert useless surveillance in non-carrier relatives. Mutational studies, ${ }^{1}$ based on conventional screening methods, have indicated that point mutations of MSH2, MLH1, or MSH6 can be detected in approximately $55 \%$ of the families, fulfilling the Amsterdam (AMS) criteria. These stipulate:

- at least three relatives with colorectal cancer, or cancer of the endometrium, small bowel, ureter, or renal pelvis

- one of whom is a first degree relative of the other two

- at least two successive generations affected

- and at least one cancer diagnosed before the age of 50 years. $^{2}$

In a recent study, we showed that genomic rearrangements of MSH2 are involved in approximately $20 \%$ of the AMS+ HNPCC families without detectable point mutations within MSH2 or MLH1. ${ }^{3}$ This study was performed using quantitative multiplex PCR of short fluorescent fragments (QMPSF), which can easily detect heterozygous genomic deletions and duplications..$^{3-7}$ This method is based on the simultaneous amplification of short genomic sequences under quantitative

Table 1 Frequency of $M S H 2$ and $M L H I$ exonic rearrangements detected by QMPSF in HNPCC families according to their status

\begin{tabular}{|c|c|c|}
\hline Status & $\mathrm{MSH} 2$ & MLHI \\
\hline AMS+ families & $120^{*}$ & $86+$ \\
\hline Without IHC‡ information & 101 & 75 \\
\hline -with a rearrangement & $16(16 \%)$ & $2(3 \%)$ \\
\hline $\begin{array}{l}\text { With selective extinction of } \\
\text { the MMR protein }\end{array}$ & 19 & 11 \\
\hline -with a rearrangement & $11(58 \%)$ & $4(36 \%)$ \\
\hline $\begin{array}{l}\text {-AMS+ families with a } \\
\text { rearrangement }\end{array}$ & $27(22 \%)$ & $6(7 \%)$ \\
\hline AMS - families & $212^{*}$ & $106+$ \\
\hline Without IHC $\mp$ information & 200 & 85 \\
\hline -with a rearrangement & $9(4 \%)$ & $2(2 \%)$ \\
\hline $\begin{array}{l}\text { With selective extinction of } \\
\text { the MMR protein§ }\end{array}$ & 12 & 21 \\
\hline -with a rearrangement & $7(58 \%)$ & $1(5 \%)$ \\
\hline $\begin{array}{l}\text {-AMS families with a } \\
\text { rearrangement }\end{array}$ & $16(8 \%)$ & $3(3 \%)$ \\
\hline
\end{tabular}

*Without $\mathrm{MSH} 2$ or $\mathrm{MLH1}$ point mutation.

†Without $\mathrm{MSH} 2$ or $\mathrm{MLH} 1$ point mutation, or $\mathrm{MSH} 2$ exonic rearrangement.

$\pm \mathrm{IHC}$ staining of the tumour.

$\S$ In the tumour.

\section{Key points}

- In hereditary non-polyposis colorectal cancer (HNPCC), point mutations of $\mathrm{MSH} 2, \mathrm{MLH1}$, or $\mathrm{MSH} 6$ are detected in approximately half of the families involved, which therefore fulfil the Amsterdam criteria (AMS).

- We analysed MSH2 in 120 AMS+ and 212 AMSHNPCC families without MSH2 or MLH1 point mutations, using quantitative multiplex PCR of short fluorescent fragments(QMPSF). We identified in 22\% of the AMS+ and in $8 \%$ of the AMS - families 19 distinct exonic deletions and two cases of duplication of $M S H 2$. We detected seven distinct $5^{\prime}$ breakpoints in the deletions removing exon 1. Specific QMPSF analysis of the MSH2 promoter in $65 \mathrm{AMS}+$ families, without MSH2/MLH1 point mutations or MSH2 exonic deletion, revealed only one case of promoter deletion. Among 86 AMS+ and 106 AMS - families, we detected seven distinct $\mathrm{MLH} 1$ exonic deletions in 7\% of the AMS + families and in 3\% of the AMS- families. We found that the selective extinction of MMR protein in the tumours was highly predictive of an MMR rearrangement.

- We conclude that $\mathrm{MSH} 2$ rearrangements are involved in at least $10 \%$ of the AMS+ families, which justifies screening for these in the routine diagnosis of HNPCC. The presence of $M L H 1$ rearrangements should be considered in AMS+ HNPCC patients, when there is a selective loss of $M L H 1$ expression in the tumours.

conditions, using dye labelled primers, and the superimposition of the electropherograms of patients and controls.

We have now integrated QMPSF into the routine diagnosis of HNPCC. We first analysed, as previously described, ${ }^{3}$ the 16 exons of MSH2 in 332 families, without point mutations within MSH2 and MLHI (table 1). These families corresponded to 120 families fulfilling AMS criteria and 212 AMSfamilies. Immunohistochemical (IHC) staining of the tumours was performed in 19 AMS+ and 12 AMS - patients, and revealed a selective extinction of the MSH2 protein. Among the AMS+ patients without IHC information, an MSH2 genomic rearrangement was detected in $16 \%$ of the cases; the detection rate reached $58 \%$ in AMS+ patients, showing a selective loss of expression of $\mathrm{MSH} 2$ in their tumours. Among the AMS - patients negative for $\mathrm{MSH} 2$ and MLH1 mutations, we found an MSH2 genomic rearrangement in $4 \%$ of the cases, when IHC analysis had not been performed, and in 58\% of the cases with IHC MSH2 extinction. QMPSF analysis of the 19 exons of MLHI was then carried out in 192 families, corresponding to 86 AMS+ 
Table 2 Summary of $M S H 2$ and $M L H 1$ exonic rearrangements detected by QMPSF in HNPCC families

\begin{tabular}{|c|c|c|c|}
\hline MSH2 & Families & MLHI & Families \\
\hline del $^{*}$ exon 1 & $\mathrm{R} 1 \dagger, \mathrm{R} 2 \dagger, \mathrm{P} 14$ & del exons 1-19 & U4, U5 \\
\hline del exons 1-2 & $\mathrm{P} 15, \mathrm{R} 12, \mathrm{R} 13, \mathrm{R} 20$ & del exon 4-6 & Lu4 \\
\hline del exons 1-4 & $\mathrm{L} 7 \dagger$ & del exon 6 & $\mathrm{~S} 10$ \\
\hline del exons 1-6 & $\begin{array}{l}\text { Li8†, R14, P16, U1, } \\
\text { U2, S1, R18 }\end{array}$ & del exons 7-9 & R19 \\
\hline del exons 1-7 & $\begin{array}{l}\text { R9t, Lu1, Lu2, } \\
\text { S2, R21 }\end{array}$ & del exons 9-10 & $\mathrm{P} 20$ \\
\hline del exons 1-8 & $\begin{array}{l}\text { R10t, R11†, } \\
\text { S3, S4 }\end{array}$ & del exon 11 & S11, U6 \\
\hline del exons $1-11$ & U3 & del exon 14 & $\mathrm{~S} 12$ \\
\hline del exons 1-15 & $\mathrm{P} 12 \dagger$ & & \\
\hline del exon 2 & S5 & & \\
\hline del exon 3 & R3†, P17 & & \\
\hline del exons 4-6 & Lu3 & & \\
\hline del exon 5 & P5† & & \\
\hline del exon 5-6 & $\mathrm{Pl3 \dagger}$ & & \\
\hline del exon 7 & $\mathrm{L6t}$ & & \\
\hline del exon 7-10 & S6 & & \\
\hline del exon 8 & $\mathrm{P} 18, \mathrm{R} 15, \mathrm{~S} 7, \mathrm{R} 16$ & & \\
\hline del exons 9-10 & P19 & & \\
\hline del exons 12-13 & $\mathrm{R} 17$ & & \\
\hline del exons 13-15 & S8 & & \\
\hline Dupł exons 7-8 & S9 & & \\
\hline Dup exons 9-10 & $\mathrm{L} 14 \dagger$ & & \\
\hline \multicolumn{4}{|c|}{$\begin{array}{l}\text { *del, deletion. } \\
\text { †Previously published in Charbonnier et al. } \\
\text { IDup, duplication. } \\
\text { We have not included in this table two } M L H 1 \text { rearrangements removing } \\
\text { exon } 2 \text { and exons } 12-13 \text { that we had initially detected by RT-PCR and } \\
\text { documented in Charbonnier, et al. }{ }^{4}\end{array}$} \\
\hline
\end{tabular}

families (including 11 families in which IHC staining of the tumours had been undertaken and revealed a selective extinction of $M L H 1)$ and 106 AMS - families. Among the AMS+ patients, we found an MLHI genomic deletion in 3\% of the cases when IHC had not been performed, and in 36\% of the cases when IHC had revealed a selective $M L H I$ extinction. Although the patients for whom IHC staining of the tumours revealed a selective loss of expression were low in number, our results indicate that the selective extinction of an $M M R$ protein within a tumour is predictive, in HNPCC families, of the genetic alteration.

We detected a total of 21 distinct $M S H 2$ exonic rearrangements, including 19 deletions and two duplications, in 43 families; and seven exonic deletions of $\mathrm{MLHI}$ in nine families (table 2). In families with an $M S H 2$ rearrangement removing exon 1, QMPSF scanning of $50 \mathrm{~Kb}$ of genomic sequences upstream of the MSH2 transcription initiation site (table 3) revealed at least seven distinct $5^{\prime}$ breakpoints. Furthermore, this analysis showed that the recurrent exonic deletions that we detected (deletions of exon 1, exons 1-2, 1-6, 1-7) had been independently generated (tables 2 and 3 ), thus excluding a founder effect. The numerous breakpoints within the $5^{\prime}$ MSH2 region led us to screen for rearrangements affecting the promoter selectively, which would have escaped the initial QMPSF analysis of MSH2 restricted to the 16 exons. We therefore performed a specific QMPSF assay for the $4.4 \mathrm{~kb}$ promoter region, using the promoter amplicons indicated in table 3, and reanalysed 65 AMS+ families without MSH2/ MLH1 point mutations or $M S H 2$ exonic deletion. We identified in a single family a $1.7 \mathrm{~kb}$ partial deletion of the promoter removing the $-1770-60$ region.

This study confirms the following.

- The frequency of MSH2 exonic rearrangements in AMS+ HNPCC families without detectable point mutations of MSH2 or MLH1 can be estimated to approximately $20 \%$ (table 1).

- We identified, on the basis of exonic and promoter rearrangements, 30 distinct genomic alterations that demonstrate the remarkable heterogeneity of $\mathrm{MSH} 2$ rearrangements (tables 2 and 3 ).

- The rearrangements that affect the MSH2 promoter selectively occur in less than $2 \%$ of AMS+ families.

- MLH1 rearrangements are involved in $7 \%$ of AMS+ families without point mutations.

Table 3 QMPSF scanning of the $5^{\prime}$ MSH2 region in HNPCC families with exonic deletions removing exon 1

\begin{tabular}{|c|c|c|c|c|c|c|c|c|c|c|}
\hline \multirow[b]{2}{*}{ Family } & \multirow[b]{2}{*}{$\begin{array}{l}\text { Exonic } \\
\text { rearrangement }\end{array}$} & \multicolumn{9}{|c|}{ QMPSF amplicons* } \\
\hline & & $\begin{array}{l}-39258 \\
-39107\end{array}$ & $\begin{array}{l}-34123 \\
-33918\end{array}$ & $\begin{array}{l}-23323 \\
-23186\end{array}$ & $\begin{array}{l}-9381 \\
-9243\end{array}$ & $\begin{array}{l}-5112 \\
-4942\end{array}$ & $\begin{array}{l}-4244 \dagger \\
-4084\end{array}$ & $\begin{array}{l}-3591 \dagger \\
-3387\end{array}$ & $\begin{array}{l}-1714 \dagger \\
-1494\end{array}$ & $\begin{array}{l}+200 \ddagger \\
327\end{array}$ \\
\hline R2 & del exon 1 & & & & & & & $+\S$ & del $\boldsymbol{\sigma}$ & del \\
\hline L7 & del exons $1-4$ & & & & & & & + & del & del \\
\hline R18 & del exons 1-6 & & & & & & & + & del & del \\
\hline U1 & del exons 1-6 & & & & & & & + & del & del \\
\hline R14 & del exons 1-6 & & & & & & + & del & del & del \\
\hline U2 & del exons 1-6 & & & & & + & del & del & / & del \\
\hline R1 & del exon 1 & & & + & del & del & del & del & del & del \\
\hline P15 & del exons 1-2 & & & + & del & del & / & del & del & del \\
\hline R9 & del exons 1-7 & & & + & del & del & del & del & del & del \\
\hline R11 & del exons 1-8 & & & + & del & del & del & del & del & del \\
\hline U3 & del exons 1-11 & & & + & del & del & / & del & / & del \\
\hline $\mathrm{R} 12$ & del exons 1-2 & & + & del & del & del & / & del & del & del \\
\hline R13 & del exons 1-2 & & + & del & I & del & / & del & I & del \\
\hline S2 & del exons $1-\overline{7}$ & + & del & del & / & del & / & del & / & del \\
\hline R10 & del exons 1-8 & + & del & del & del & del & del & del & del & del \\
\hline Li8 & del exons 1-6 & del & del & del & del & del & del & del & del & del \\
\hline S1 & del exons 1-6 & del & / & del & I & del & I & del & 1 & del \\
\hline Lul & del exons 1-7 & del & / & del & / & del & / & del & / & del \\
\hline Lu2 & del exons 1-7 & del & / & del & / & del & / & del & / & del \\
\hline S3 & del exons 1-8 & del & / & del & / & del & / & del & / & del \\
\hline S4 & del exons 1-8 & del & / & del & / & del & / & del & / & del \\
\hline P12 & del exons 1-15 & del & del & del & del & del & del & del & del & del \\
\hline
\end{tabular}

*Numbered from the MSH2 transcription initiation site (- 68 bp from the ATG) according to the chromosome 2.

†These amplicons correspond to the MSH2 promoter defined by lwahashi, et al (1998).

$\ddagger$ Amplicon corresponding to exon 1.

§Non-deleted.

- Deleted.

Working draft sequence (contig NT_034483). Primers and QMPSF conditions are available upon request. 
Two recent papers, ${ }^{8-9}$ have reported higher detection rates of $\mathrm{MSH} 2$ and $\mathrm{MLH1}$ rearrangements, respectively, but these remarkable percentages are probably due to the differences between the populations analysed. The first study, ${ }^{8}$ performed on 24 AMS+ families without point mutations, using Southern Blot analysis, documented the detection of an MSH2 genomic deletion in 50\% of the cases. This remarkable detection rate is probably explained by the fact that the exons 1-6 deletion, detected in seven families, were shown to be associated to a founder effect. ${ }^{8}$ The second study, ${ }^{9}$ based on the QMPSF analysis of 52 AMS+ families without point mutations, reported the detection of genomic deletions of MSH 2 and MLHI in $12 \%$ of the families, for each gene. In this work, the existence of a common haplotype, in four families harbouring a deletion of $M L H 1$ exons $1-10$, suggested a founder effect and may also have led to an overestimation of the relative contribution of $M L H 1$ deletions in HNPCC.

In conclusion, we recommend that investigation for $\mathrm{MSH} 2$ rearrangements be included systematically in the routine diagnosis of HNPCC. The contribution of these alterations to HNPCC is higher than that of MSH6 mutations. ${ }^{10-12}$ Considering the lower frequency of MLHI rearrangements, except in certain populations where they are associated with a founder effect, ${ }^{8-9}$ it is probably more efficient to search in HNPCC families only when IHC staining of the tumours has revealed a selective loss of $M L H I$ expression.

\section{ACKNOWLEDGEMENTS}

We are grateful to Mario Tosi for critical review of the manuscript, and to the clinicians who referred HNPCC families.

\section{Authors' affiliations}

F Di Fiore, F Charbonnier, C Martin, S Frerot, T Frebourg, Department of Genetics, University Hospital, and INSERM EMI 9906 -IFRMP, Faculty of Medicine, Rouen, France

S Olschwang, C Boisson, INSERM U434 and Saint Antoine Hospital, Paris, France

Q Wang, Department of Molecular Oncology, Centre Léon Bérard, Lyon, France

M-P Buisine, Laboratory of Biochemistry and Molecular Biology, University Hospital, Lille, France

M Nilbert, Department of Oncology, University Hospital, Lund, Sweden A Lindblom, Department of Clinical Genetics, Karolinska Hospital, Stockholm, Sweden

This work was supported by L' Association pour la Recherche sur le Cancer, and La Ligue Nationale de Lutte contre le Cancer.

Correspondence to: Dr T Frebourg, Department of Genetics, University Hospital, and INSERM EMI 9906-IFRMP, Faculty of Medicine, $22 \mathrm{Bd}$. Gambetta, 76183 Rouen, France; Frebourg@chu-roven.fr
Received 14 July 2003

Accepted 16 August 2003

\section{REFERENCES}

1 Peltomäki P, Vasen H. The International Collaborative Group on Hereditary Nonpolyposis Colorectal Cancer. Mutations predisposing to hereditary nonpolyposis colorectal cancer: database and results of a collaborative study, Gastroenterology 1997;113:1146-58.

2 Vasen HF, Watson P, Mecklin JP, Lynch HT. New clinical criteria for hereditary nonpolyposis colorectal cancer (HNPCC, Lynch syndrome) proposed by the International Collaborative Group on HNPCC. Gastroenterology 1999;116:1453-6

3 Charbonnier F, Olschwang S, Wang Q, Boisson C, Martin C, Buisine MP, Puisieux A, Frebourg T. MSH2 in contrast to $M L H 1$ and $M S H 6$ is frequently inactived by exonic and promoter rearrangements in hereditary nonpolyposis colorectal cancer. Cancer Res 2002;62:848-53.

4 Charbonnier F, Raux G, Wang Q, Drouot N, Cordier F, Limacher JM, Saurin JC, Puisieux A, Olschwang S, Frebourg T. Detection of exon deletions and duplications of the mismatch repair genes in hereditary nonpolyposis colorectal cancer families using multiplex polymerase chain reaction of short fluorescent fragments. Cancer Res 2000;60:2760-3.

5 Casilli F, Di Rocco ZC, Gad S, Tournier I, Stoppa-Lyonnet D, Frebourg T, Tosi M. Rapid detection of novel BRCA1 rearrangements in high-risk breastovarian cancer families using multiplex PCR of short fluorescent fragments. Hum Mut 2002;20:218-26.

6 Bougeard G, Charbonnier F, Moerman A, Martin C, Ruchoux MM, Drouot N Frebourg T. Early onset brain tumor and lymphoma in $\mathrm{MSH} 2$ deficient children. Am J Hum Genet 2003;72:213-16.

7 Bougeard G, Brugieres L, Chompret A, Gesta P, Charbonnier F, Valent A, Martin C, Raux G, Feunteun J, Bressac-de Paillerets B, Frebourg T. Screening for TP53 rearrangements in Li-Fraumeni syndrome reveals a complete deletion of the TP53 gene. Oncogene 2003;22:840-6.

8 Wagner A, Barrows A, Wijnen JT, Van Der Klift H, Franken PF, Verkuijlen P, Nakagawa H, Geugien M, Jaghmohan-Changur S, Breukel C, MeijersHeijboer H, Morreau H, Van Puijenbroek M, Burn J, Coronel S, Kinarski Y, Okimoto R, Watson P, Lynch JF, De La Chapelle A, Lynch HAT, Fodde R. Molecular analysis of hereditary nonpolyposis colorectal cancer in the United States: high mutation detection rate among clinically selected families and characterization of an American founder genomic deletion of the $\mathrm{MSH} 2$ gene. Am J Hum Genet 2003;72:1088-100.

9 Wang Y, Friedl W, Lamberti C, Jungck M, Mathiak M, Pagenstecher C, Propping P, Mangold E. Hereditary nonpolyposis colorectal cancer: frequent occurrence of large genomic deletions in $\mathrm{MSH} 2$ and $\mathrm{MLH} 1$ genes. Int $J$ Cancer 2003;103:636-41.

10 Wijnen J, de Leeuw W, Vasen $\mathrm{H}$, Van Der Kliff H, Moller $\mathrm{P}$, Stormorken A, Meijers-Heijboer $H$, Lindhout D, Menko F, Vossen S, Moslein G, Tops C, Brocker-Vriends A, Wu Y, Hofstra R, Sijmons R, Cornelisse C, Morreau H, Fodde R. Familial endometrial cancer in female carriers of MSH6 germline mutations. Nat Genet 1999;23:142-4.

11 Huang J, Kuismanen SA, Liu T, Chadwick RB, Johnson CK, Stevens MW, Richards SK, Meek JE, Gao X, Wright FA, Mecklin JP, Jarvinen HJ, Gronberg H, Bisgaard ML, Lindblom A, Peltomaki P. MSH6 and MSH3 are rarely involved in genetic predisposition to nonpolypotic colon cancer. Cancer Res 2001;61:1619-23.

12 Berends MJW, Wu Y, Sijmons RH, Mensik RGJ, van der Sluis T, HordijkHos JM, de Vries EGE, Hollema H, Karrenbeld A, Buys CHCM, van der Zee AGJ, Hofstra RMW, Kleibeuker JH. . Molecular and clinical characteristics of $\mathrm{MSH} 6$ variants: an analysis of 25 index carriers of a germline variant. Am J Hum Genet 2002;70:26-37.

13 Iwahashi Y, Ito E, Yanagisawa Y, Akiyama Y, Yuasa Y, Onodera T, Maruyama K. Promoter analysis of the human mismatch repair gene hMSH2. Gene 1998;213:141-7.

\section{CORRECTION}

The authors of the paper by Howell et al in the September issue (HRPT2 mutations are associated with malignancy in sporadic parathyroid tumours. J Med Genet 2003;40:657-63) have notified us of an error. In figure 1, third row from the bottom, for Family ID (Family F1)4†, the histology of the tumour should be Adenoma and not Carcinoma. The authors apologise for the error. 